\title{
REPRESENTATION OF THE PROJECTABLE AND STRONGLY PROJECTABLE HULLS OF A LATTICE-ORDERED GROUP ${ }^{1}$
}

\author{
DONALD A. CHAMBLESS
}

\begin{abstract}
A representable $l$-group $G$ can be embedded into a projectable (strongly projectable) l-group; an essential extension of $G$ which is minimal with respect to being projectable (strongly projectable) is unique. In this paper these projectable and strongly projectable "hulls" of $G$ are represented using direct limits. If $G$ is an $f$-ring, or $f$-ring without nonzero nilpotent elements, then so are these hulls.
\end{abstract}

1. Introduction. An l-group $G$ is said to be representable if it can be embedded into a cardinal product of totally ordered groups, projectable if each polar subgroup of the form $g^{\prime \prime}$ for $g \in G$ is a cardinal summand of $G$, and strongly projectable if every polar subgroup of $G$ is a cardinal summand. If $G$ is projectable then each polar subgroup of $G$ is a normal subgroup and so $G$, and hence each $l$-subgroup of $G$, is representable [4, Theorem 1.8]. Conversely, if $G$ is representable then $G$ is a dense $l$ subgroup of its orthocompletion [2, pp. 116, 125] which is strongly projectable.

If $G$ is a large $l$-subgroup of a strongly projectable (projectable) $l$-group $K$ then the intersection $H$ of all strongly projectable (projectable) $l$-subgroups of $K$ containing $G$ is strongly projectable (projectable), and Conrad [5] has shown that $H$ is the unique (up to $l$-isomorphism extending the identity map of $G$ ) minimal strongly projectable (projectable) essential extension of $G$, and called $H$ the sp-hull (p-hull) of $G$. In this note we wish to give direct limit representations of these hulls in a fashion similar to that in which Conrad [3, pp. 455-457] has represented the orthocompletion of $G$. References containing related material are [1], [2], [5], [7].

Notation. We use the notation and terminology of the general references [4], [6] unless otherwise specified. $G$ will always denote a

Received by the editors June 24, 1971.

AMS 1970 subject classifications. Primary 06A55; Secondary 06A70.

Key words and phrases. Strongly projectable l-group, projectable l-group, representation of $l$-groups, representable $l$-group, direct limits of $l$-groups.

1 This paper represents a part of the author's dissertation submitted to the Mathematics Department of the Graduate School of Tulane University. The author would like to express his appreciation to Professor P. F. Conrad for his assistance and direction.

(c) American Mathematical Society 1972 
representable $l$-group (written additively, but not assumed to be abelian). For a subset $S$ of $G, S^{\prime}$ denotes the polar of $S$, and $\mathscr{P}(G)$ the complete Boolean algebra of polar subgroups of $G . \prod A_{\lambda}$ denotes the cardinal product of the l-groups $A_{\lambda}$ and $A \oplus B$ denotes the cardinal sum of the l-groups $A, B$.

2. Representation of the hulls. The following discussion of the direct limit of a directed system of $l$-groups and $l$-isomorphisms and construction of the orthocompletion of $G$ was given by Conrad in [3], but is repeated here for completeness.

Let $\Lambda$ be a lower directed set and suppose $\left\{G_{\lambda}, \Pi_{\alpha \beta}, \Lambda\right\}$ is a lower directed system of $l$-groups and $l$-isomorphisms (i.e., if $\alpha \geqq \beta$ then $\Pi_{\alpha \beta}$ is an $l$-isomorphism of $G_{\alpha}$ into $G_{\beta}$ where the usual transitive laws are satisfied). If $\boldsymbol{P}$ is the set product of the $G_{\lambda}$, let $L$ denote the collection of all $t \in \boldsymbol{P}$ such that if $\alpha \geqq \beta$ then $t_{\alpha} \neq 0$ or $t_{\beta}=0$ implies $t_{\alpha} \Pi_{\alpha \beta}=t_{\beta}$, and $t_{\alpha}=0$ and $t_{\beta} \neq 0$ implies $t_{\beta} \notin G_{\alpha} \Pi_{\alpha \beta}$.

Let $\theta$ denote the 0 element of $\boldsymbol{P}$, and for $\theta \neq k, t \in L$ select $\alpha$ such that $k_{\alpha} \neq 0 \neq t_{\alpha}$; then $k+t$ is defined by $(k+t)_{\alpha}=k_{\alpha}+t_{\alpha}$ (each nonzero component of a nonzero element of $L$ completely determines that element). Also call $t \in L$ positive if $t=\theta$ or $t_{\alpha}>0$ for some $\alpha$. Then $L$ is an $l$-group and the direct limit of the directed system above; if $t \in L$ and $t_{\alpha}$ is not comparable with 0 then $(t \vee \theta)_{\alpha}=t_{\alpha} \vee 0$.

Call a maximal disjoint subset of $\mathscr{P}(G)$ a partition and let $D(G)$ denote the set of all partitions of $\mathscr{P}(G)$. If $\mathscr{A}$ and $\mathscr{C}$ are partitions then we write $\mathscr{A} \leqq \mathscr{C}(\mathscr{A}$ refines $\mathscr{C})$ if $A \in \mathscr{A}$ implies $A \subseteq C$ for some $C \in \mathscr{C}$. If $\mathscr{B} \in D(G)$, let $\mathscr{D}=\{C \cap B \neq 0 \mid C \in \mathscr{C}, B \in \mathscr{B}\}$ (the intersection of $\mathscr{B}$ and $\mathscr{C}$ ); then $\mathscr{D} \leqq \mathscr{B}, \mathscr{C}$ and so $D(G)$ is a lower directed set. If $C \in \mathscr{C}$ and $\left\{A_{\gamma}\right\}_{\Gamma}$ is the collection of all elements of $\mathscr{A}$ included in $C$ then $C^{\prime}=\bigcap A_{\gamma}^{\prime}$ and so the natural map of $G / C^{\prime}$ into $\Pi\left\{G\left|A_{\gamma}^{\prime}\right| \gamma \in \Gamma\right\}$ is an $l$-isomorphism. In this way a natural $l$-isomorphism $\Pi_{\mathscr{C} \mathscr{A}}$ of $G_{\mathscr{C}}=\Pi\left\{G / C^{\prime} \mid C \in \mathscr{C}\right\}$ into $G_{\mathscr{A}}$ is determined, and $\left\{G_{\mathscr{C}}, \Pi_{\mathscr{C} \mathscr{A}}, D(G)\right\}$ is then a directed system; let its direct limit be $L$. If $t \in L$ and $\mathscr{C} \in D(G)$ we write $t_{\mathscr{C}}=\left(\cdots, t(C)+C^{\prime}, \cdots\right)$ where $C \in \mathscr{C}$ and $t(C) \in G$ (the $t(C)$ are only determined mod $C^{\prime}$, of course). If $g \in G$ and $\mathscr{B} \in D(G)$ let $\tilde{g}(B)=g$ for all $B \in \mathscr{B}$; then the map $g \rightarrow \tilde{g}$ is an $l$-isomorphism of $G$ onto a dense $l$-subgroup $\tilde{G}$ of $L$. $L$ is the orthocompletion of $G$ in the sense of Bernau [2] (see [3, pp. 455-457]).

Now to construct the sp-hull of $G$ let $F(G)$ denote the set of all finite elements of $D(G)$ and consider the directed system $\left\{G_{\mathscr{C}}, \Pi_{\mathscr{C}, \mathscr{L}}, F(G)\right\}$. If $S$ is the direct limit of this system then we have a natural $l$-isomorphism $g \rightarrow \tilde{g}$ of $G$ into $S$ given by $\tilde{g}(B)=g$ for all $B \in \mathscr{B}$ and all $\mathscr{B} \in F(G)$, and $\tilde{G}$ is a dense $l$-subgroup of $S$. Therefore the map $Q \rightarrow Q \cap \tilde{G}$ determines a natural Boolean isomorphism of $\mathscr{P}(S)$ onto $\mathscr{P}(G)[3$, p. 455], and so if 
$P \in \mathscr{P}(G)$ and $*$ denotes the polar operation in $S$, then $\tilde{G} \cap \tilde{P}^{* *}=\tilde{P}$ (henceforth we will identify $G$ and $\widetilde{G}$ ).

If $\theta \neq f \in S, P \in \mathscr{P}(G)$, and $\mathscr{B} \in F(G)$ refines $\left\{P, P^{\prime}\right\}$ then $f \in P^{*}$ if and only if $f(B)=0 \bmod B^{\prime}$ for all $B \in \mathscr{B}$ included in $P$. Similarly, $f \in P^{* *}$ if and only if $f(B)=0 \bmod B^{\prime}$ for each $B \in \mathscr{B}$ included in $P^{\prime}$. The proofs of these facts are routine.

THEOREM A. $S$ is the sp-hull of $G$.

Proof. Let $Q \in \mathscr{P}(S)$ and $\theta \neq t \in S ; Q$ is of the form $P^{* *}$ for a unique $P \in \mathscr{P}(G)$. Pick $\mathscr{B} \in F(G)$ such that $\mathscr{B}$ refines $\left\{P, P^{\prime}\right\}$ and $t_{\mathscr{B}} \neq 0$, and define $k \in S$ by specifying that, for all $B \in \mathscr{B}, k(B)=t(B)$ if $B \subseteq P$, and $k(B)=0$ otherwise. Then $k \in P^{* *}=Q$ and $t-k \in P^{*}=Q^{*}$, so that $t=k+$ $(t-k) \in Q \oplus Q^{*}$. Therefore $S$ is a strongly projectable essential extension of $G$.

Now suppose that $G \subseteq H \subseteq S$ where $H$ is strongly projectable and let $\perp$ denote the polar operation in $H$. Assume that $t \in H, Q \in \mathscr{P}(H)$, and write $t=t_{1}+t_{2}$ in $Q \oplus Q^{\perp}=H$ and $t=k_{1}+k_{2}$ in $Q^{* *} \oplus Q^{*}=S$. Since $Q \subseteq Q^{* *}$ and $Q^{\perp} \subseteq Q^{*}$ it follows that $t_{1}=k_{1}$ and $t_{2}=k_{2}$; thus the projections $t_{1}$ of $t$ into $Q$ in $H$ and $k_{1}$ of $t$ into $Q^{* *}$ in $S$ are identical.

Now let $\theta \neq t \in S$ with $0 \neq t_{\mathscr{C}}=\left(g_{1}+C_{1}^{\prime}, \cdots, g_{n}+C_{n}^{\prime}\right)$ and let $t_{j} \in S$ be determined by $t_{j}\left(C_{j}\right)=g_{j}$ and $t_{j}\left(C_{i}\right)=0$ for $i \neq j, 1 \leqq j \leqq n$. Then $t_{j}$ is the projection of $g_{j}$ into $C_{j}^{* *}$, and since $g_{j} \in H$ it follows as discussed in the last paragraph that $t_{j}$ is also the projection of $g_{j}$ into $C_{j}^{\perp \perp}$. Thus $t_{j} \in H$ and so $t=t_{1}+\cdots+t_{n} \in H$. Hence $H=S$, and so $S$ is a minimal strongly projectable essential extension of $G$.

REMARK. If $A$ is an $f$-ring then each polar subgroup of $A$ is an ideal and so any directed system of the $A_{\mathscr{C}}$ and $\Pi_{\mathscr{C} \mathscr{A}}$ is a system of $f$-rings and $l$-ring isomorphisms. Thus the direct limit of such a system is also an $f$-ring. If $A$ has no nonzero nilpotent elements then $A / P$ also has none for each polar subgroup $P$ of $A$, and hence such a direct limit also has this property.

In particular, the sp-hull $S$ of $A$ is an $f$-ring (with no nonzero nilpotent elements if $A$ has none), and the map $g \rightarrow \tilde{g}$ is an $l$-ring isomorphism of $A$ into $S$.

The p-hull of $G$ can be constructed in an analogous (but somewhat more complicated) fashion. Here let $\boldsymbol{T}$ denote the sublattice of $\mathscr{P}(G)$ generated by all polars of the form $g^{\prime \prime}$ or $g^{\prime}$ for $g \in G$ and let $T(G)$ be the set of all $\mathscr{C} \in F(G)$ which are subsets of $T$. This gives rise to a directed system $\left\{G_{\mathscr{C}}, \Pi_{\mathscr{C} \mathscr{S}}, T(G)\right\}$, the direct limit of which we denote by $T$. Again we have a natural embedding $g \rightarrow \tilde{g}$ given by $\tilde{g}(C)=g$ for all $C \in \mathscr{C}$ and all $\mathscr{C} \in T(G)$ and we denote the polar operations in $G$ and $T$ by ' and * respectively. We identify $G$ and $\tilde{G}$. 
LEMMA. If $\theta \neq k \in T$ with $0 \neq k_{\mathscr{C}}=\left(\cdots, k_{j}+C_{j}^{\prime}, \cdots\right)$ then $k^{* *}=$ $\bigvee\left(C_{j}^{* *} \cap k_{j}^{* *}\right)$ where the sup occurs in $\mathscr{P}(T)$.

Proof. Without loss of generality $k>\theta$. Assume first that $\mathscr{C}=\left\{C_{j}\right\}_{1}^{n}$ and $0 \neq k_{\mathscr{C}}=\left(g+C_{1}^{\prime}, 0, \cdots, 0\right)$. If $\mathscr{D}$ is the intersection of $\mathscr{C}$ and $\left\{g^{\prime \prime}, g^{\prime}\right\}$ then $0 \neq k_{\mathscr{D}}=\left(g+\left(C_{1} \cap g^{\prime \prime}\right)^{\prime}, 0, \cdots, 0\right)$ and so $k \in\left(C_{1} \cap g^{\prime \prime}\right)^{* *}=C_{1}^{* *} \cap g^{* *}$. We wish to show that if $\theta<t \in C_{1}^{* *} \cap g^{* *}$ then $t \wedge k>\theta$; then it will follow that $k^{* *}=C_{1}^{* *} \cap g^{* *}$, as desired.

Consider $\theta<t \in C_{1}^{* *} \cap g^{* *}$ and let $t_{\mathscr{B}}>0$ for some $\mathscr{B}$ refining $\mathscr{D}$. Then $t(B)=0 \bmod B^{\prime}$ when $B \in \mathscr{B}$ is not included in $C_{1} \cap g^{\prime \prime}$, and if $B \subseteq C_{1} \cap g^{\prime \prime}$ and $g \notin B^{\prime}$ then $g+B^{\prime}$ is a weak unit for $G / B^{\prime}$. Therefore if $t(B), g \notin B^{\prime}$ for some $B \in \mathscr{B}$ then $t_{\mathscr{B}} \wedge k_{\mathscr{B}}>0$ and so $\theta<t \wedge k$ as desired. Suppose by way of contradiction that for all $B \in \mathscr{B}, g \notin B^{\prime}$ implies $t(B) \in B^{\prime}$. Then $g_{\mathscr{B}} \wedge t_{\mathscr{B}}=0$ and so $t \in g^{*}$. Hence $\theta<t \in g^{*} \cap g^{* *}=\theta$, a contradiction. It follows then that $t \wedge k>\theta$ and so $k^{* *}=C_{1}^{* *} \cap g^{* *}$.

In the general case $\theta<k$ and $0<k_{\mathscr{C}}=\left(k_{1}+C_{1}^{\prime}, \cdots, k_{n}+C_{n}^{\prime}\right)$. If $\theta \leqq t_{j} \in T$ is determined by $\left(t_{j}\right)_{\mathscr{G}}=\left(0, \cdots, 0, k_{j}+C_{j}^{\prime}, 0, \cdots, 0\right)$, then

$$
t_{j}^{* *}=k_{j}^{* *} \cap C_{j}^{* *} \text { and } k=\bigvee t_{j}
$$

so that $k^{* *}=\bigvee t_{j}^{* *}=\bigvee\left(k_{j}^{* *} \cap C_{j}^{* *}\right)$.

THEOREM B. Tis the p-hull of $G$.

Proof. Suppose $\theta \neq k, f \in T$ and $0 \neq k_{\mathscr{C}}=\left(k_{1}+C_{1}^{\prime}, \cdots, k_{n}+C_{n}^{\prime}\right)$. Then by the lemma $k^{* *}=\bigvee\left(C_{j}^{* *} \cap k_{j}^{* *}\right)$, and so if $k^{* *} \cap G=P \in \mathscr{P}(G)$ then $P=\bigvee\left(G \cap C_{j}^{* *} \cap k_{j}^{* *}\right)=\bigvee\left(C_{j} \cap k_{j}^{\prime \prime}\right)$. Thus $P \in T$ and hence $\left\{P, P^{\prime}\right\} \in T(G)$. Pick $\mathscr{B} \in T(G)$ refining $\left\{P, P^{\prime}\right\}$ such that

$$
0 \neq f_{\mathscr{B}}=\left(f_{1}+B_{1}^{\prime}, \cdots, f_{m}+B_{m}^{\prime}\right),
$$

and let $t \in T$ be specified by $t\left(B_{j}\right)=f_{j}$ if $B_{j} \subseteq P$ and $t\left(B_{j}\right)=0$ otherwise. Then $f=t+(f-t) \in P^{* *} \oplus P^{*}=k^{* *} \oplus k^{*}$, and so it follows that $T$ is projectable.

Now suppose that $G \subseteq H \subseteq T$ where $H$ is projectable and let $\perp$ denote the polar operation in $H$. If $h \in H$ then $h^{\perp} \subseteq h^{*}$ and $h^{\perp \perp} \subseteq h^{\perp \perp * *}=h^{* *}$ [5, (5i)]; thus if $k \in H$ then the projections of $k$ into $h^{\perp \perp}$ in $H$ and into $h^{* *}$ in $T$ coincide.

Assume now that $\theta<t \in T$ with $t_{\mathscr{C}}>0$.

Case 1. Suppose first that $t_{\mathscr{C}}=\left(g+C_{1}^{\prime}, 0, \cdots, 0\right)$ where without loss of generality $0<g \notin C_{1}^{\prime}$. Since $C_{1} \in T$ we have $C_{1}=\bigvee_{I} \wedge_{J} S_{i j}$ where $I$ and $J$ are finite sets and $S_{i j}$ has the form $g_{i j}^{\prime \prime}$ or $g_{i j}^{\prime}$ for some $g_{i j} \in G$. Let $\mathscr{B}$ be the intersection of all the partitions $\left\{S_{i j}, S_{i j}^{\prime}\right\}$; we claim that $\mathscr{B}$ refines $\left\{C_{1}, C_{1}^{\prime}\right\}$. For if $B \in \mathscr{B}$ and $B \nsubseteq C_{1}$, then for each $i$ there is a $j_{i}$ such that $B \nsubseteq S_{i j_{i}}$. Then $B \subseteq S_{i j_{i}}^{\prime}$ and so $B \subseteq \bigwedge_{I} \bigvee_{J} S_{i j}^{\prime}=C_{1}^{\prime}$. 
Now let $t_{i j} \in T$ be determined by $t_{i j}(B)=g$ if $B \subseteq S_{i j}$ and $t_{i j}(B)=0$ otherwise, and let $k=\bigvee_{I} \wedge_{J} t_{i j}$. If $k(B) \neq 0 \bmod B^{\prime}$ then $B \subseteq C_{1}$, while if $B \subseteq C_{1}$ then $B \nsubseteq C_{1}^{\prime}$ and so it follows that $B \subseteq \wedge_{J} S_{i j}$ for some $i$, and hence $k(B)=g=t(B)$. Therefore $t_{\mathscr{B}}=k_{\mathscr{B}}$ and so $t=\bigvee_{I} \bigwedge_{J} t_{i j}$. Since $t_{i j}$ is the projection of $g \in H$ into $S_{i j}^{* *}=g_{i j}^{* *}$ or $g_{i j}^{*}$, we have $t_{i j} \in H$; thus $t \in H$.

Case 2. In the general case we have $t=t_{1}+\cdots+t_{n}$ where the $t_{j}$ have the form considered in Case 1. Thus $t_{j} \in H$ and so $t \in H$. Therefore $H=T$, and so $T$ is a minimal projectable essential extension of $G$.

REMARK. It follows as before that if $A$ is an $f$-ring, or an $f$-ring with no nonzero nilpotent elements, then so is the p-hull of $A$.

\section{REFERENCES}

1. I. Amemiya, A general spectral theory in semi-ordered linear spaces, J. Fac. Sci. Hokkaido Univ. Ser. I. 12 (1953), 111-156. MR 15, 137.

2. S. Bernau, Orthocompletion of lattice groups, Proc. London Math. Soc. (3) 16 (1966), 107-130. MR 32 \#5554.

3. P. Conrad, The lateral completion of a lattice-ordered group, Proc. London Math. Soc. (3) 19 (1969), 444-480. MR 39 \#5442.

4. - Lattice-ordered groups, Tulane University, New Orleans, La., 1970.

5. - The hulls of representable l-groups and f-rings, J. Austral. Math. Soc. (to appear).

6. L. Fuchs, Partially ordered algebraic systems, Pergamon Press, Oxford; AddisonWesley, Reading, Mass., 1963. MR 30 \#2090.

7. T. Speed, On lattice-ordered groups, J. Austral. Math. Soc. (to appear).

Department of Mathematics, University of Florida, Gainesville, Florida 32601 